\title{
Tubular Phononic Crystal Sensor
}

A. Gueddida ${ }^{1}$, Y. Pennec $*^{1}$, V. Zhang ${ }^{1}$, F. Lucklum ${ }^{2}$, M. Vellekoop ${ }^{3}$, N. Mukhin ${ }^{4}$, R. Lucklum ${ }^{4}$, B. Bonello ${ }^{5}$, B. Djafari Rouhani ${ }^{1}$.

\footnotetext{
${ }^{1}$ Institut d'Electronique, Microélectronique et Nanotechnologie, UMR CNRS8520, Université de Lille, 59650 Villeneuve d'Ascq, France

${ }^{2}$ Centre for Acoustic-Mechanical Microsystems (CAMM), Technical University of Denmark (DTU), 2800 Kgs. Lyngby,Denmark

${ }^{3}$ Institute for Microsensors, -actuators and -systems (IMSAS), Microsystems Center Bremen (MCB), University of Bremen, D-28359 Bremen, Germany

${ }^{4}$ Otto-von-Guericke-University Magdeburg, Institute for Micro and Sensor Systems, D39016, Germany

${ }^{5}$ Sorbonne Université, UPMC Université Paris 06 (INSP-UMR CNRS 7588), 4 place Jussieu 75005 Paris, France
}

*corresponding author: yan.pennec@univ-lille.fr

\begin{abstract}
We propose the design of a tubular phononic crystal (TPC) for the purpose of sensing the physical properties of a liquid filling the hollow part of the tube. The TPC is constituted by a periodic repetition of washers along a hollow pipe with the advantage of avoiding any perturbation of a flowing fluid by any element inside the tube. Using finite element simulations, we demonstrate the existence of complete as well as polarization dependent bandgaps inside which one can design localized modes associated with defects. The most sensitive cavity to the liquid sound velocity is found to be constituted by a Fabry-Perot (F-P) cavity. The signature of the cavity modes can be detected as peaks or dips in the transmission spectrum as well as at the external surface of the cavity. We study the dramatic effect of the liquid viscosity, more particularly shear viscosity, on these features and discuss the conditions for their practical observation. A TPC test sample made of a polymer is fabricated by means of $3 \mathrm{D}$ printing and characterized without the liquid by transmission measurements. The comparison with the simulations showed the necessity of considering the damping of the polymer whose effect on the transmission features is discussed. Our sensor design can find many applications at different scales in several systems transporting a fluid, as microfluidic channels in micro and nanotechnology, syringe in medicine, or pipe lines in civil engineering.
\end{abstract}

\section{Introduction}

Phononic crystals are periodic materials known for their ability to exhibit forbidden bands inside which acoustic or elastic waves are prohibited from propagation. The phononic crystals are now well-known for their ability to guide, control and manipulate the acoustic and elastic waves $^{1-4}$. These physical properties are generally based on the existence of defect modes 
inside the band gaps that are confined in cavities or linear waveguides. This capacity to control the propagation of elastic waves has opened up a broad field of applications, depending on the targeted frequency.

In the last decade, phononic crystals have seen a growing interest in the field of sensing, in particular as an innovative resonant platform for investigating the volumetric properties of liquids ${ }^{5}$. These properties include material parameters such as mass density, sound velocity or viscosity and their evolution under the variation of temperature or the concentration in a mixture solution. The liquid can be introduced as a component of the phononic crystal or inside a small cavity localized in the perfect structure. In the latter case, the phononic cavity mode appears in the transmission spectrum as narrow peaks or dips, depending on their occurrence respectively inside or outside the bandgap. The sensing functionality can be achieved through the phononic crystal by enhancing the intrinsic solid/liquid interaction. It has been shown ${ }^{5}$ that the cavity modes can exhibit both high quality factors, up to $\mathrm{Q} \approx 4 \times 10^{3}$, and high sensitivity (for instance of the order of $S \approx 122 \mathrm{~Hz} / \mathrm{m}_{\mathrm{s}} \mathrm{s}^{-1}$ in the $200 \mathrm{kHz}$ range in ref. 6) to the speed of sound despite the small amount of liquid, thus enabling a powerful tool for measuring the sound velocity of the liquid ${ }^{5,6}$. The performance of the sensor is then estimated in relation to several parameters of practical interest like the temperature ${ }^{7}$, the concentration of a compound in a mixture ${ }^{8}$ or the phase transition of the analyte ${ }^{9}$. Of course, many other techniques and structures have addressed the acoustic characterization of materials without the utilization of periodic crystals. To name a few, let us mention the traditional quartz crystal microbablance $^{10}$, the liquid sensor applications of MEMS resonators ${ }^{11}$, the utilization of NEMS for ultra-low mass detection ${ }^{12}$, a waveguide sensor for the measurement of viscosity in highly viscous liquid ${ }^{13}$ or an interferometric technique for the measurement of Young's modulus in small size samples ${ }^{14}$. In this paper, we focus on the specific case of phononic crystals where the acoustic wave does not probe only the interfacial layer between the solid resonator and the liquid but penetrates the whole liquid volume, thereby inspecting the acoustic properties of its entire volume.

The utilization of phononic crystals as liquid sensors was first proposed by R. Lucklum et al. ${ }^{15}$ who have shown the feasibility of the concept considering a 1D multilayered structure and 2D phononic crystal with holes where one component is a liquid. The purpose was to estimate quantitatively the potential of a phononic crystal to be an efficient tool for sensing. Two general conditions must be met in order to achieve this objective. Firstly, the transmission spectrum should display well-defined features, secondly these features are highly sensitive to 
the acoustic velocity of the fluid to be analyzed. High performance designs have been demonstrated by several authors who introduced cavities ${ }^{16,17}$, slots ${ }^{18,19}$ or waveguides ${ }^{20}$ in 1D or $2 \mathrm{D}$ periodic crystals. The acoustic wave, confined in a liquid cavity, produces one or more localized modes with high sensitivity to the sound velocity of the liquid. Another concept is based on acoustic ring resonator employing a two-dimensional surface phononic crystal, which has been proposed to analyze binary gas mixtures facing the ring resonator with highsensitivity ${ }^{21}$.A last requirement is the achievement of a high-quality factor of the characteristic feature, i.e., peak or dip in the transmission or reflection spectrum in order to achieve a high resolution of the frequency measurement and to keep successive peaks or dips separated from each other. Finally, another physical mechanism exploited as sensor is based on the extraordinary acoustic transmission as underlying phenomenon. The acoustic wave, normally impinging to the periodic perforated plate couples with a mode involving the liquid in the holes and the nearest neighborhood. We have shown that the mechanism behind extraordinary acoustic transmission involves volumetric liquid as well as structural vibrations of solid-liquid composites. At resonance, the volumetric vibrations are substantially supported by plate vibrations and combine in compound bi-medium oscillators ${ }^{22}$. The respective peak frequency has been exploited to sense acoustic properties of the embedding fluid with high sensitivity ${ }^{23-}$ 25

The recent development of phononic crystal sensors is going toward integration by dealing with plates of finite thicknesses as proposed by Jin et al. ${ }^{26}$ who investigated phononic crystal plates with hollow pillars. By filling the hollow part with a liquid, it has been shown, theoretically ${ }^{26}$ and later experimentally ${ }^{27}$, that the resulting localized modes were sensitive to the height of the liquid in the hole, its concentration, or temperature. Very recently, Bonhomme et al. ${ }^{28}$ extended further the application range of phononic crystals with the detection of the mass of nanoparticles. Finally, the introduction of the concept of phoXonic crystals based on the simultaneous confinement of phonon and photon in the same cavity opened the possibility of a dual characterization of the acoustic and optical refractive index of the liquid ${ }^{19,25,29-32}$.

In practice, in various systems useful for engineering applications the liquids to be monitored are transported by pipes, and the aforementioned phononic crystal sensors cannot be implemented directly inside these systems. The objective of this paper is to study a TPC, consisting of a periodic arrangement of cylindrical washers distributed along a hollow tube ${ }^{33}$ where the liquid can flow. Few papers have dealt with the manipulation of acoustic waves in 1D corrugated cylindrical waveguide. The existence of Bragg gap in a periodic composite 
material pipe has been demonstrated theoretically ${ }^{34}$ and experimentally ${ }^{35}$ with the goal of reducing and controlling the elastic vibrations of the pipe. A pipe filled with air can also carry the acoustic pressure generated by a loudspeaker that can be modulated with the internal structuration of the rigid solid part ${ }^{36,37}$. Xiao et al. ${ }^{38}$ demonstrated the existence of topologic states at the interface of two phononic crystals having different topological band gap characteristics.

In this paper, we study a TPC, made of polymer, and consisting of a periodic arrangement of cylindrical washers along a hollow tube (see section 1). By filling the pipe with a liquid, and due to the low impedance contrast between the liquid and the solid, we will investigate the propagation of the acoustic pressure in the liquid, the elastic vibrations in the solid and their interaction. One specificity of the proposed design is that the periodic elements are only attached in the external part of the pipe and do not perturb the flow of the liquid inside the tube. We investigate the effect of mass density and sound velocity variations on the transmission of acoustic waves through a cavity, by focusing on a mixture of water and 2propanol with varying concentrations. Considering a Fabry-Perot type cavity, we show the existence of well-defined peaks and dips in the transmission spectrum associated with the confined modes of the cavity. However, we demonstrate that the quality factors of these features can be strongly altered by the liquid viscosity, and in particular by its shear (or dynamic) component. While the effect of viscosity has been considered in some previous works dealing with solid-liquid phononic crystals ${ }^{6,18}$, we give here a detailed analysis of the critical role of each viscosity component, while the considered cavity modes are more complex than in a simple planar cavity. Therefore, we discuss the optimal conditions for the observation of the defect modes in the process of liquid characterization.

In section 2, we study the physical properties of the TPC and the solid-fluid interaction by means of dispersion and transmission curves. In section 3, the sensing property of the TPC is discussed through the insertion of a Fabry-Perot type cavity in the middle of the TPC. Section 4 is dealing with the effect of liquid and material dissipation on the sensing performance of the structure. Finally, we present in a Supplementary Information (SI) section some preliminary experiments, before liquid filling, with a few samples of TPC fabricated with the help of a 3D printer. The conclusions are drawn in section 5.

\section{Tubular phononic crystal}

As shown in Figure 1(a), the TPC is defined as a hollow tube with a periodic arrangement of cylindrical washers. The geometrical parameters involved in the TPC are the lattice constant 
$a$, the outer and inner radii of the tube, called $R$ and $r$, respectively. The washers are defined by their width $w$ and height $h$ above the outer radius $R$. The liquid to be monitored is located in the hollow part of the tube, indicated by the green color in the schematic representation. The TPC is made of a polymer, defined by the Young modulus $E=3.2 \mathrm{GPa}$, Poisson's ratio $v=0.35$ and mass density $\rho=1190 \mathrm{kgm}^{-3}$. The results will be illustrated for a set of waterpropanol mixtures (molar ratio, $x$, of 2-propanol from 0 to 59.6\%) for which the sound velocities and mass densities are listed in table 1 from experimental measurements in the literature ${ }^{15,39}$. A peculiarity of these mixtures is a non-monotonous variation of the acoustic velocity, which displays a maximum, whereas the mass density decreases continuously. The dispersion and transmission curves calculations have been performed by means of the software COMSOL Multiphysics, based on the finite element method.

(a)

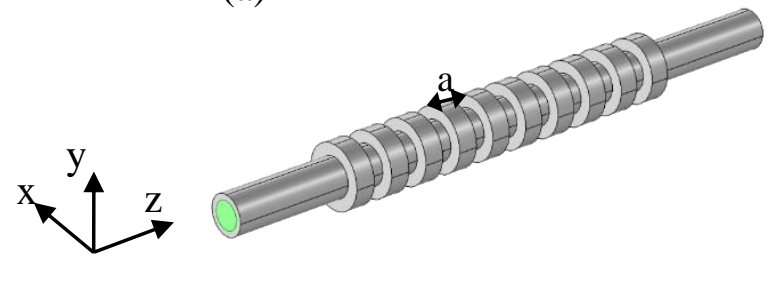

(b)

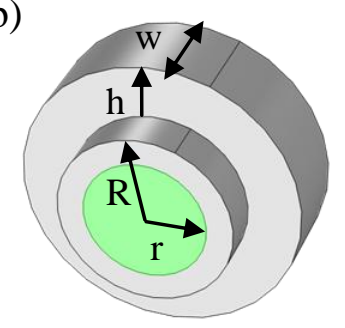

Figure 1: (a) 3D schematic representation of the tubular phononic crystal made of polymer and filled with water (green). (b) 3D view of the elementary unit cell used for the calculation of the dispersion curves. Periodic boundary conditions are applied on the front and back sides of the hollow tube.

Table 1 : Mass density and speed of sound of 2-propanol-water mixtures with different molar ratio, $x$, of propanol from experimental measurements in the literature ${ }^{9,33}$.

\begin{tabular}{ccc}
\hline Molar ratio x & Mass density $\left(\mathbf{k g} / \mathbf{m}^{\mathbf{3}}\right)$ & Speed of sound $\mathbf{c}_{\mathbf{l}}(\mathbf{m} / \mathbf{s})$ \\
\hline $\mathbf{0}$ & 1000 & 1490 \\
$\mathbf{0 . 0 2 1}$ & 990 & 1545 \\
$\mathbf{0 . 0 5 6}$ & 974 & 1588 \\
$\mathbf{0 . 1 5 8}$ & 933 & 1472 \\
$\mathbf{0 . 3 4 7}$ & 881 & 1367 \\
$\mathbf{0 . 5 9 6}$ & 841 & 1298 \\
\hline
\end{tabular}

To calculate the dispersion curves, one considers an elementary unit cell of the TPC (Fig. 1(b)) on which the Bloch-Floquet periodic conditions are applied in the direction of 
periodicity, z. Boundary conditions on the continuity of the normal component of the displacement field and normal stresses are applied at the solid/liquid interface. Figure 2(a) represents the dispersion curves of the TPC filled with water, where the blue, red and black branches correspond to the longitudinal, flexural and torsional modes ${ }^{35}$, respectively. Starting from $\Gamma(q=0)$, one can note the presence of two longitudinal branches, $\boldsymbol{L}_{\boldsymbol{l}}$ and $\boldsymbol{L}_{\boldsymbol{2}}$, a flexural, $\boldsymbol{F}$, and a torsional, $\boldsymbol{T}$. With the set of geometrical parameters, $a=1 \mathrm{~mm}, r=0.35 \times a, R=$ $0.5 \times a, w=0.5 \times a$ and $h=0.3 \times a$, we find an absolute phononic band gap around $350 \mathrm{kHz}$ represented by the hatched grey area. The large phononic band gap has been achieved after an optimization of the geometrical parameters of the water filled TPC following a systematic study (see Appendix). We do not present the higher torsional modes which appear only above $600 \mathrm{kHz}$.

The transmission curves have been calculated considering a finite crystal composed of tenunicells' (Fig. 1(a)). The elastic waves are generated by applying a force, loaded at the inlet of the TPC, and probed at the outlet, in both cases far from the first and last washers. The excitation is done in the solid part as described in reference ${ }^{40}$. To avoid any unwanted reflected wave from the boundaries of the TPC, perfect matched layers (PML) conditions are added at both ends of the structures. This geometry is convenient for a direct analysis of the transmission spectra and localized cavity modes in the TPC. However, for the purpose of making the excitation and detection in such a way as to not perturb the flow of the liquid in the TPC, another configuration based on a circumferentially placed phase array transducers ${ }^{40}$ would be more convenient. In the next section, we shall also propose a detection of the wave at the level of the cavity in the TPC which can be based on the same configuration. For the sake of a simpler physical than technical discussion, we did not implement this configuration in our simulations. Figures 2(b) and 2(c) represent the transmission curves calculated for the longitudinal (blue) and the flexural (red) polarizations. The transmission spectrum associated with the longitudinal excitation shows the occurrence of two band gaps separated by a narrow pass band, whereas the transmission spectrum obtained for the flexural excitation shows a large band gap around $350 \mathrm{kHz}$ and a narrow band gap around $550 \mathrm{kHz}$. A good agreement is found between the transmission pass bands and the dispersion branches of the same polarization. 
(a)

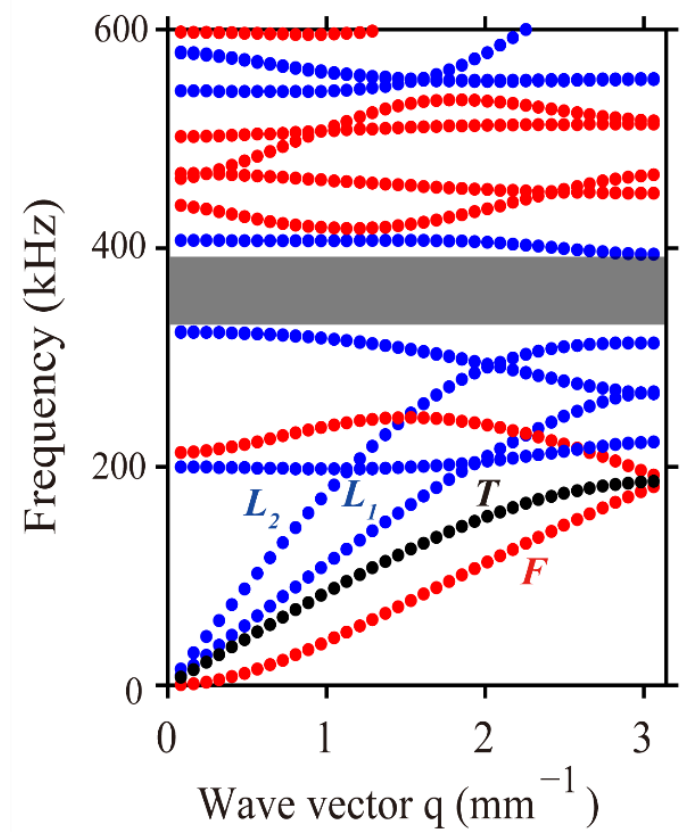

(b)

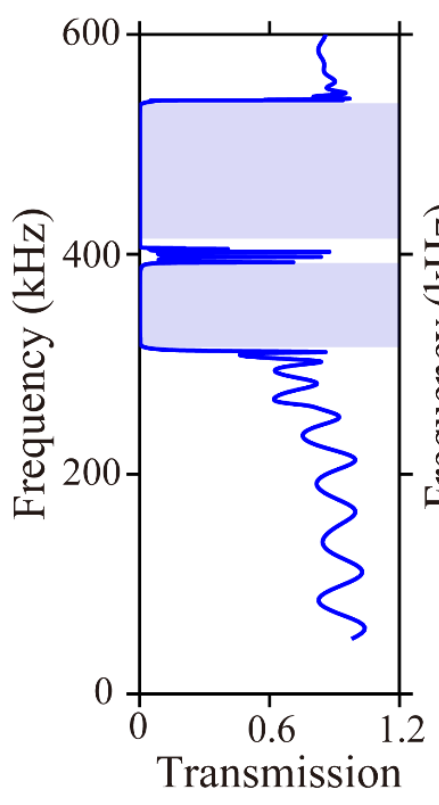

(c)

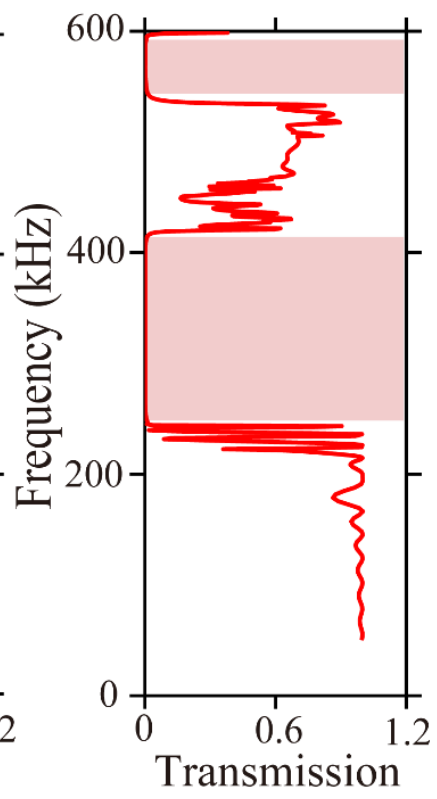

Figure 2: (a) Dispersion curves of the perfect tubular phononic crystal. The blue, red and black colors correspond to the longitudinal, flexural and torsional modes. (b) and (c) Transmission curves for longitudinal (blue) and flexural (red) polarization of the incident wave. The hatched areas represent the band gaps.

(a)

$$
0 \stackrel{\mathrm{U}_{\text {Liquid }} / \mathrm{U}_{\text {Total }}}{ } 1
$$

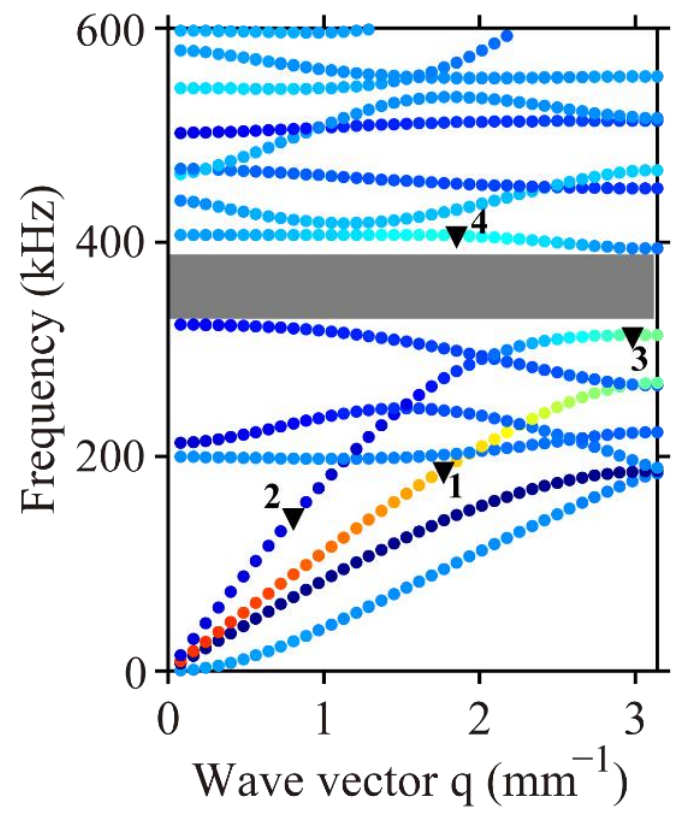

(b)

\section{Liquid+Solid Liquid}

4

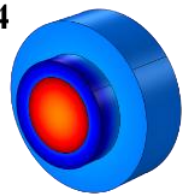

3

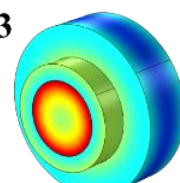

2

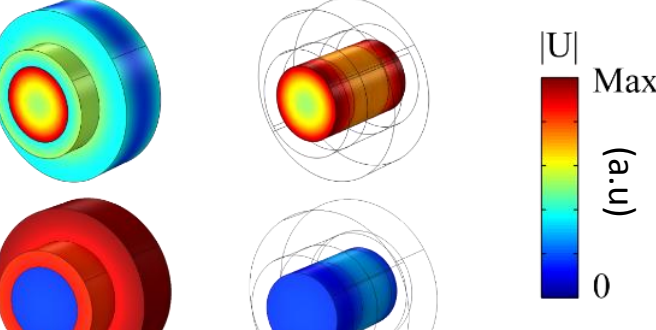

1

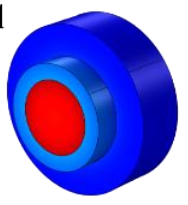

$|\mathrm{U}|$

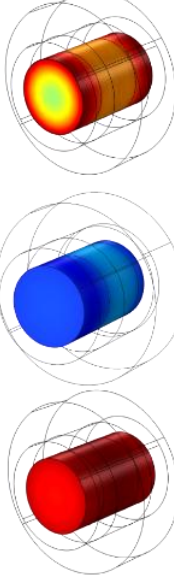

$\operatorname{Max}$

(1)

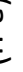

Figure 3: (a) Dispersion curves of the tubular phononic crystal colored according to the ratio between the average displacement field in the liquid and in the whole unit cell. (b) Maps of the displacement field associated with the modes indicated by the black triangles on the dispersion curves. 
To analyze the distribution of the displacement field over the unit cell and discriminate the modes according to their confinements in the liquid or solid parts of the structure, we proceeded to a band sorting calculation (Fig. 3(a)), namely evaluating the ratio of the average displacement in the liquid as compared to the whole unit cell.

$$
P=\frac{\iiint_{\text {Liquid }}\left(\left|u_{x}\right|^{2}+\left|u_{y}\right|^{2}+\left|u_{z}\right|^{2}\right) d v}{\iiint_{\text {Unit cell }}\left(\left|u_{x}\right|^{2}+\left|u_{y}\right|^{2}+\left|u_{z}\right|^{2}\right) d v}
$$

Figure 3(a) represents the result through a coloration of the TPC dispersion curves where the red and blue colors mean a strong localization in the liquid (e.g. branch 1) and in the solid (e.g. branch 2), respectively, whereas the branches with the intermediate light blue and green colors (e.g. branches 3 and 4) are associated with the modes whose displacement field is shared between the two constituents. Figure 3(b) illustrates the displacement fields corresponding to four selected modes. Note that the fields in the fluid and in the solid should be presented with the same scale in this analysis. Because the propagation of the acoustic waves in the liquid is described by the acoustic pressure, the acoustic displacement field components are deducted from the pressure according to:

$$
\boldsymbol{u}=\frac{1}{\rho \omega^{2}} \nabla p
$$

Where $\boldsymbol{u}$ is the displacement field vector in the liquid, $\rho$ and $\omega$ represent the mass density of the liquid and the angular frequency, respectively, and $p$ the acoustic pressure.

From Fig. 3(b), one can notice that the two longitudinal branches $L_{1}$ and $L_{2}$ are mainly confined in the liquid and in the solid. For modes 3 and 4, the field is distributed between both the liquid and the solid, which allows to expect an interaction between the two components.

\section{Tubular phononic crystal sensor}

As mentioned in the introduction, the strategy adopted to design the TPC sensor is to avoid any perturbation of the fluid flow inside the tube. Therefore, the periodic elements (washers) are added to the external part of the tube only. Accordingly, the information on the nature of the liquid should be extracted from the solid part, either at the outlet of the tube or on the external surface of the tube. Furthermore, a defect (cavity) is introduced in the center of the TPC. Defect modes should display a strong coupling between the solid and the liquid when having the sensor application in mind. This can be tested for the longitudinal branches 3 and 4 
of the phononic crystal by changing the characteristics of a washer in the TPC center. We have noticed that the sensitivity of the localized modes associated with such defects remained low with respect to the liquid properties. Therefore, we adopted a different type of defect consisting of a Fabry-Perot (F-P) cavity of sufficient length as explained below (Fig. 4) in order to induce localized modes with both high-quality factors and a strong coupling with the solid material through the transverse component of the displacement field. First, the choice of the F-P cavity is dictated by the possibility of stationary waves which are expected to provide highly confined modes. A minimal length of the cavity is necessary to bring these modes inside the band gaps where they can fully take benefit of the localization properties of the phononic crystals and display high quality factors; otherwise the F-P modes will appear above the bandgaps. Moreover, by increasing the length of the cavity, the stationary F-P oscillations offer many orders of vibration that can display both longitudinal and transverse components of the displacement field which is helpful to enhance the interaction between the liquid and the solid. This is necessary to make the phononic crystal sensitive to the acoustic properties of the liquid while allowing an easy detection of the confined modes from the external surface of the tube.

The geometrical parameters of the design are based on a systematic study directed to the occurrence of well-defined features in the transmission spectrum with both high-quality factors, $Q$, and sensitivity to the characteristic acoustic properties of the liquid. Figure 4 shows a 2D section of the TPC containing the Fabry-Perot cavity of total length $L_{c}=3 \mathrm{~mm}$. With the aim of enhancing the solid/liquid interaction inside the cavity, we decreased the outer radius of the tube to $R_{c}=0.4 \mathrm{~mm}$ over a length of $2 \mathrm{~mm}$, instead of $R=0.5 \mathrm{~mm}$ everywhere else, while keeping the inner radius equal to $r=0.35 \mathrm{~mm}$ to not alter the flow of the liquid inside the pipe. The height of the washers has been kept to $h=0.3 \mathrm{~mm}$ to insure the same band gaps as obtained in the perfect TPC. The transmission is investigated with two different polarizations of the excitation, i.e. longitudinal (Fig. 5) and flexural (Fig.7).

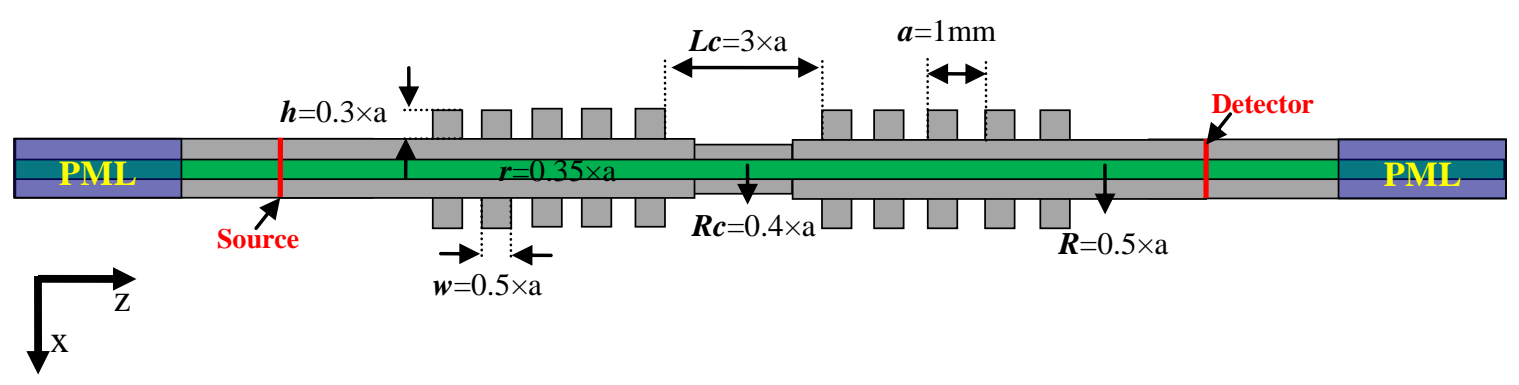


Figure 4: 2D schematic view of the TPC with a Fabry-Perot cavity. The green color corresponds to the liquid flowing in the pipe.

Figure 5(a) represents the transmission curves obtained for a longitudinal polarization through the TPC including the F-P cavity, where the blue hatched areas correspond to the partial band gaps associated with the longitudinal excitation when the tube is filled with water. One can notice a series of well-defined peaks and dips in the transmission spectrum associated with the confined modes of F-P cavity. These modes give rise to peaks or dips depending on whether their frequencies fall inside the band gaps or the pass bands.

To illustrate the aforementioned sensing properties, we have calculated the transmission curves when the pipe is filled either with pure water (solid line) or with a mixture of 2propanol and water with molar ratio of 59.6\% (dashed line), as examples of liquids listed in table $1^{15,39}$. Among the peaks and dips, we have selected two modes that display the highest sensitivity to the liquid properties. These are the peak A and dip B when the filling liquid is water that move respectively to $\mathrm{A}^{\prime}$ and $\mathrm{B}^{\prime}$ when changing water by the water-propanol mixture with lower acoustic velocity and mass density than water. It is also worth noting that the positions of the band gaps are also altered when the filling liquid is changed from water (as shown in Fig.5(a)), but these modifications are weaker than the shift of F-P modes. In Fig. 5(b), we depict the displacement fields associated to modes A and B. The color bar in Fig.5(b) has been adapted to represent the displacement field at the same scale in the liquid and in the solid. In both cases, the modes correspond to well confined stationary waves inside the F-P cavity and display high $Q$-factors. In general, one can note a higher quality factor of the peaks than of the dips. However, these effects are also significantly affected by the frequency position of the peak or dip with respect to the band edges. Indeed, the peaks and dips can become broader and lose their quality factors when approaching the edges of the bandgap. Finally, it is again worth noting that the acoustic fields of these modes are spread in both the solid and liquid constituents; therefore, they can be measured both in the transmission signal (Fig. 5) or at the outer surface of the tube in the cavity area as it will be discussed in section 4 and Fig. 10. 
(a)

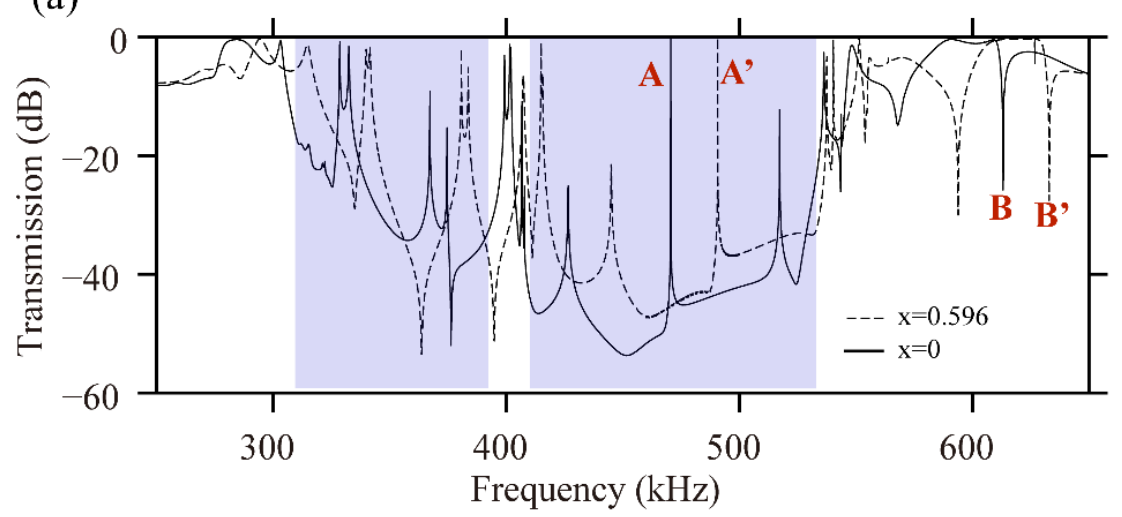

(b)
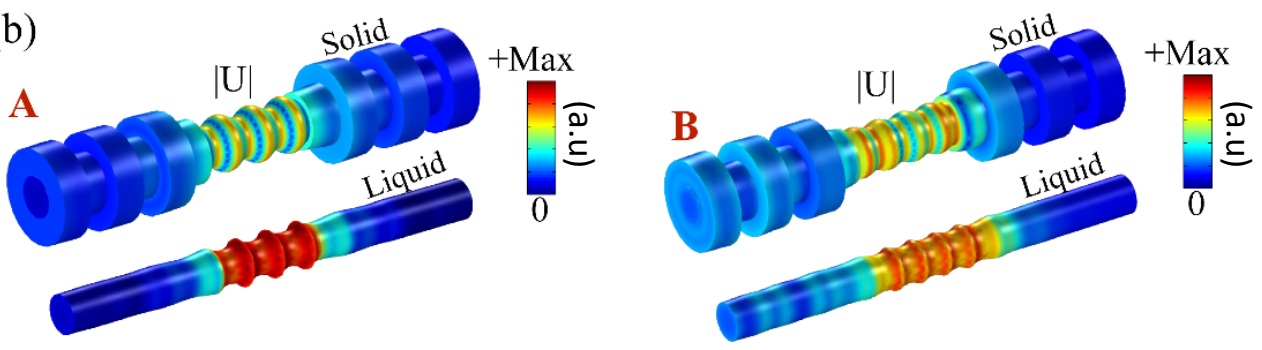

(c)
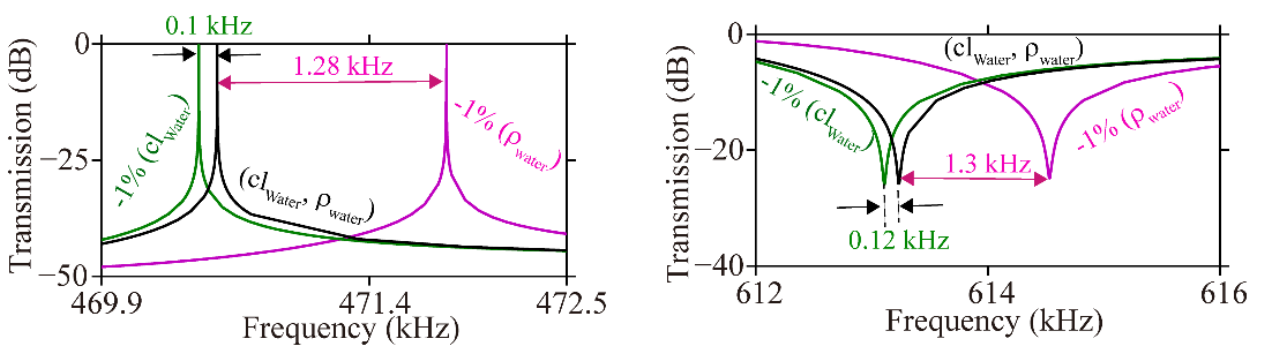

(d)
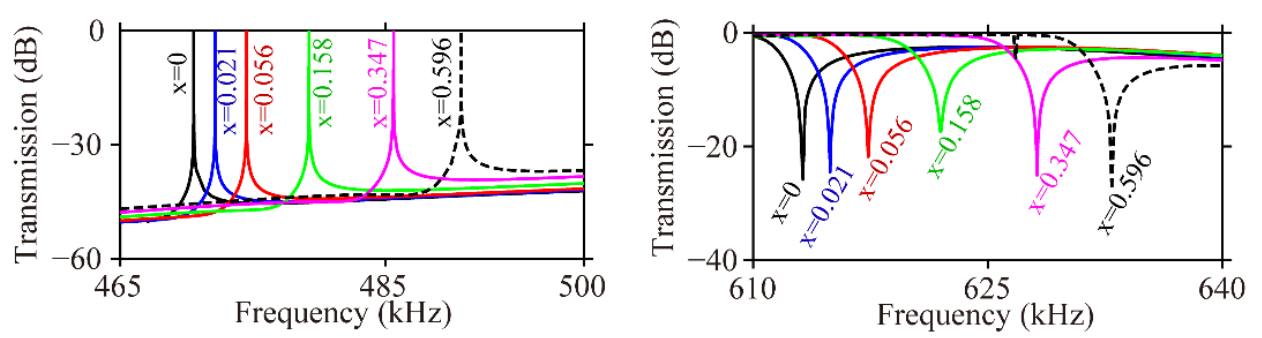

Figure 5: (a) Transmission curves of the TPC containing the F-P cavity for a longitudinal excitation, when the tube is filled with water (solid line) or a mixture of water and 2-propanol at $x=59.6 \%$ (dashed line). The blue areas correspond to the position of the partial band gaps when the tube is filled with water. (b) Maps of the displacement field at the frequencies of peak A and dip B in the solid (left panel) and in the fluid (right panel). (c) Shift of the peak A (left panel) and dip B (right panel) assuming a change either in the mass density (pink curves) or in the acoustic velocity (green curves) of water by $1 \%$. (d) Same as (c) for the set of propanol-water mixtures with molar ratio $x$ ranging from 0 to $59.6 \%$. 
Two complementary illustrations of these results are presented in Figs. 5(c) and (d). First, starting from the water as the liquid filling the TPC, we arbitrarily change either the velocity or the mass density of the liquid by $1 \%$. We show in Fig. 5(c) the corresponding variations for the peak A (left panel) and dip B (right panel). These results show that the features in the transmission spectrum are sensitive to both the acoustic velocity $c_{l}$ and the mass density $\rho$ which are the two independent physical parameters of the liquid. It can be noted that for the polymer material chosen for the TPC, the sensitivity is stronger to $\rho$ than to $c_{l}$ which is in contrast to the observation reported in some other sensor designs ${ }^{15}$. More quantitatively, one can define the sensitivities $S_{c}$ and $S_{\rho}$ as the frequency shifts of the cavity modes for a small variation of either velocity $c_{l}$ or mass density $\rho$

$$
S_{c}=\frac{\Delta f}{\Delta c_{l}}\left(H z m^{-1} s\right) \quad S_{\rho}=\frac{\Delta f}{\Delta \rho_{l}}\left(\mathrm{Hzkg}^{-1} \mathrm{~m}^{3}\right) \quad \text { Eq. } 3
$$

Another interesting quantity is the so-called figure of merit $(F o M)$, given by:

$$
F o M_{c}=\frac{S_{c} \times Q_{i}}{f_{i}}(\mathrm{~m} / \mathrm{s})^{-1} \quad F_{o} M_{\rho}=\frac{S_{\rho} \times Q_{i}}{f_{i}} \mathrm{~kg}^{-1} \mathrm{~m}^{3} \quad \text { Eq. } 4
$$

where $Q_{i}$ is the quality factor of the cavity mode $I$ and defined by the ratio of the frequency $f_{i}$ to the Full-Width at Half Maximum (FWHM) frequency difference of the peak or dip. FoM provides information whether two very close features can be distinguished.

Table 2 summarizes the different characteristics, namely frequency, quality factor, sensitivities and FoMs, associated with the peak A and dip B in Fig.5(c). As noticed previously, the cavity modes are more sensitive to variation of the mass density than to the speed of sound and, the $Q$-factor and $F o M$ of the peak A are higher than those associated with the dip B.

\begin{tabular}{|c|c|c|c|c|}
\hline & \multicolumn{2}{|c|}{ Peak A } & \multicolumn{2}{|c|}{ Dip B } \\
\hline Frequency $(\mathbf{k H z})$ & \multicolumn{2}{|c|}{470.54} & \multicolumn{2}{|c|}{613.23} \\
\hline \multirow[t]{2}{*}{ Sensitivity } & $\mathrm{S}_{\mathrm{c}}\left(\mathrm{Hz} \mathrm{m}^{-1} \mathrm{~s}\right)$ & $\mathrm{S}_{\rho}\left(\mathrm{Hz} \mathrm{kg}^{-1} \mathrm{~m}^{3}\right)$ & $\mathrm{S}_{\mathrm{c}}\left(\mathrm{Hz} \mathrm{m}^{-1} \mathrm{~s}\right)$ & $\mathrm{S}_{\rho}\left(\mathrm{Hz} \mathrm{kg}^{-1} \mathrm{~m}^{3}\right)$ \\
\hline & 6.9 & 128 & 8.4 & 129 \\
\hline Quality factor & \multicolumn{2}{|c|}{$3 \times 10^{6}$} & \multicolumn{2}{|c|}{$1.5 \times 10^{3}$} \\
\hline \multirow{2}{*}{$\begin{array}{l}\text { Figure of Merit } \\
\qquad(\text { FoM })\end{array}$} & $\operatorname{FoM}_{c}(\mathrm{~m} / \mathbf{s})^{-1}$ & $\operatorname{FoM}_{\rho}\left(\mathrm{kg}^{-1} \mathrm{~m}^{3}\right)$ & $\operatorname{FoM}_{c}(\mathrm{~m} / \mathrm{s})^{-1}$ & $\operatorname{FoM}_{\rho}\left(\mathrm{kg}^{-1} \mathrm{~m}^{3}\right)$ \\
\hline & 44 & 816 & 0.02 & 0.31 \\
\hline
\end{tabular}

Table 2: Sensor characteristics calculated for peak A and dip B, longitudinal polarization 
In Fig. 5(d), we show the evolution of the peak A (left panel) and dip B (right panel) for a set of 2-propanol-water mixtures with molar fraction $x$ ranging from 0 to $59.6 \%$. We note that all concentrations can be distinguished.

Fig. 6 presents the evolution of sensor sensitivity, $S_{i}$, and frequency, $f_{i}$, for peak A and dip B as a function of $x$ considering the literature values of sound velocity and density of the liquid mixture at the respective molar ratio. Here, the sensitivity has been determined between two successive values of $x$ as $\mathrm{S}=\Delta f / \Delta x$ where $\Delta f$ is the frequency shift caused by the change in the propanol concentration. Since both the sensitivities of peak A and dip B and the respective frequencies are monotonic functions with respect to $x$ one can conclude the dominating effect of liquid density. In the lower panel we present the dependence of mass density and speed of sound as a function of the molar ratio $x$ of the 2-propanol-watermixture. Obviously, the initial increase in the speed of sound giving rise to a maximum is overcompensated by the continuous decrease in the mass density.
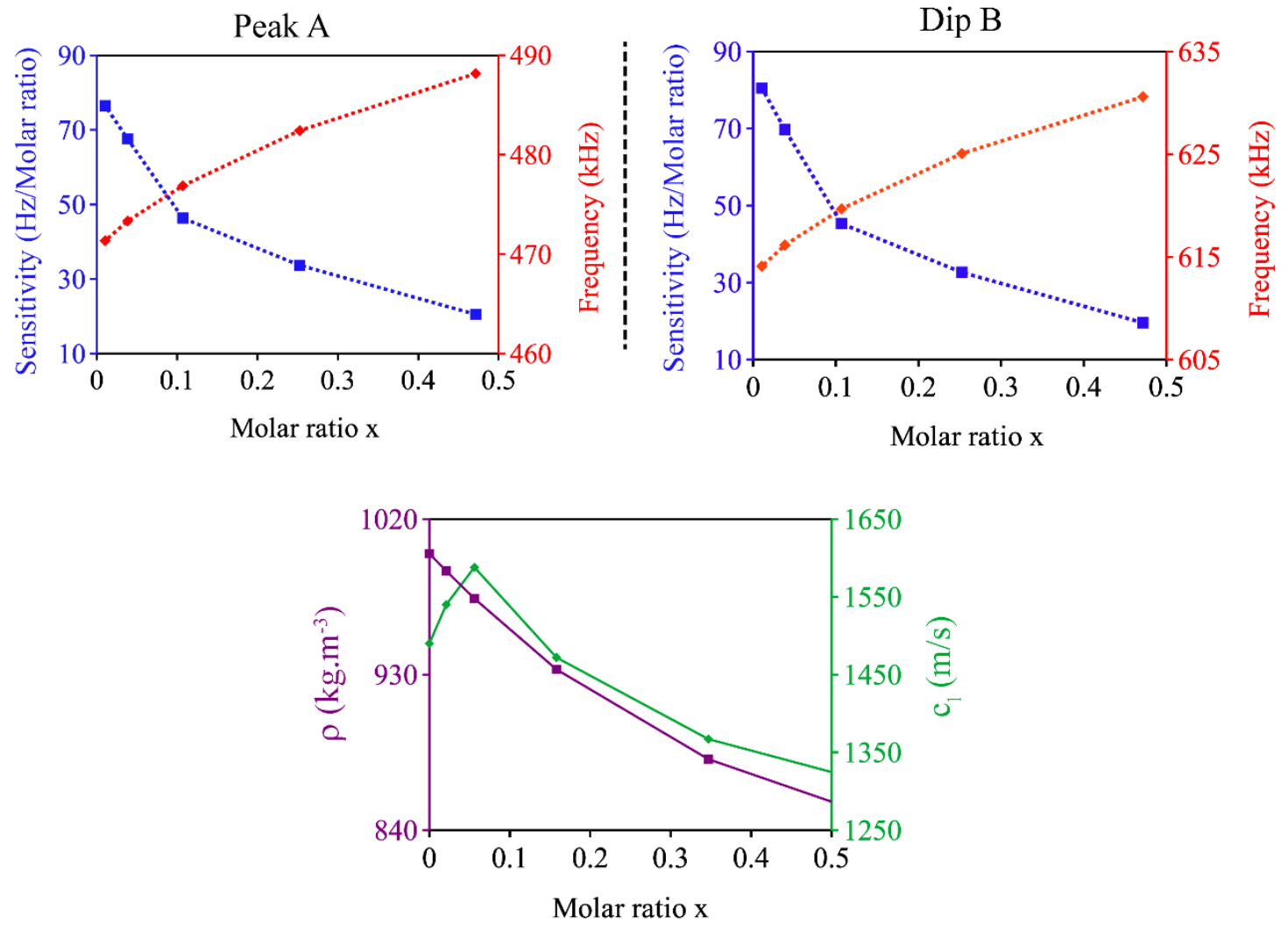

Figure 6: Sensitivity and frequency of the peak A (left panel) and dip B (right panel) as a function of the molar ratio of the 2-propanol in water. The lower panel shows the evolution of the mass density and the speed of sound as a function of the molar ratio taken from experimental measurements in the literature $e^{9,33}$. 
Next, we consider the case of an incident wave of flexural polarization. Fig. 7 presents results similar to those shown in Fig. 5. As in the previous case, the F-P cavity provides dips and peaks in the transmission curves (Fig. 7(a)), depending on whether the mode falls in a pass band or in the band gaps (red hatched areas). Figure 7(b) shows the maps of the displacement field associated with the peak C and dip D. In both cases, the flexural wave is trapped inside the cavity, giving rise to F-P oscillations. Owing to the nature of the flexural excitation, the displacement field is symmetric with respect to $\mathrm{yOz}$ plane while being antisymmetric with respect to $\mathrm{xOz}$. The mode $\mathrm{C}$ is transmitted through the TPC while the mode $\mathrm{D}$ is reflected back to the entrance. The two modes are well confined in the cavity. It means that the sensitivity to the liquid can be detected both at the exit and at the outer surface of the cavity. Fig. 7(c) shows the sensitivity and frequency of the peak C and dip D when changing either the mass density or the acoustic velocity of water by $1 \%$. As we noticed for the longitudinal excitation, the peak $\mathrm{C}$ and $\operatorname{dip} \mathrm{D}$ are more sensitive to the variations in mass density than in speed of sound. Finally, Fig. 7(d) shows the evolution of peak C and dip D when filling the TPC with a set of propanol-water mixtures. 
(a)
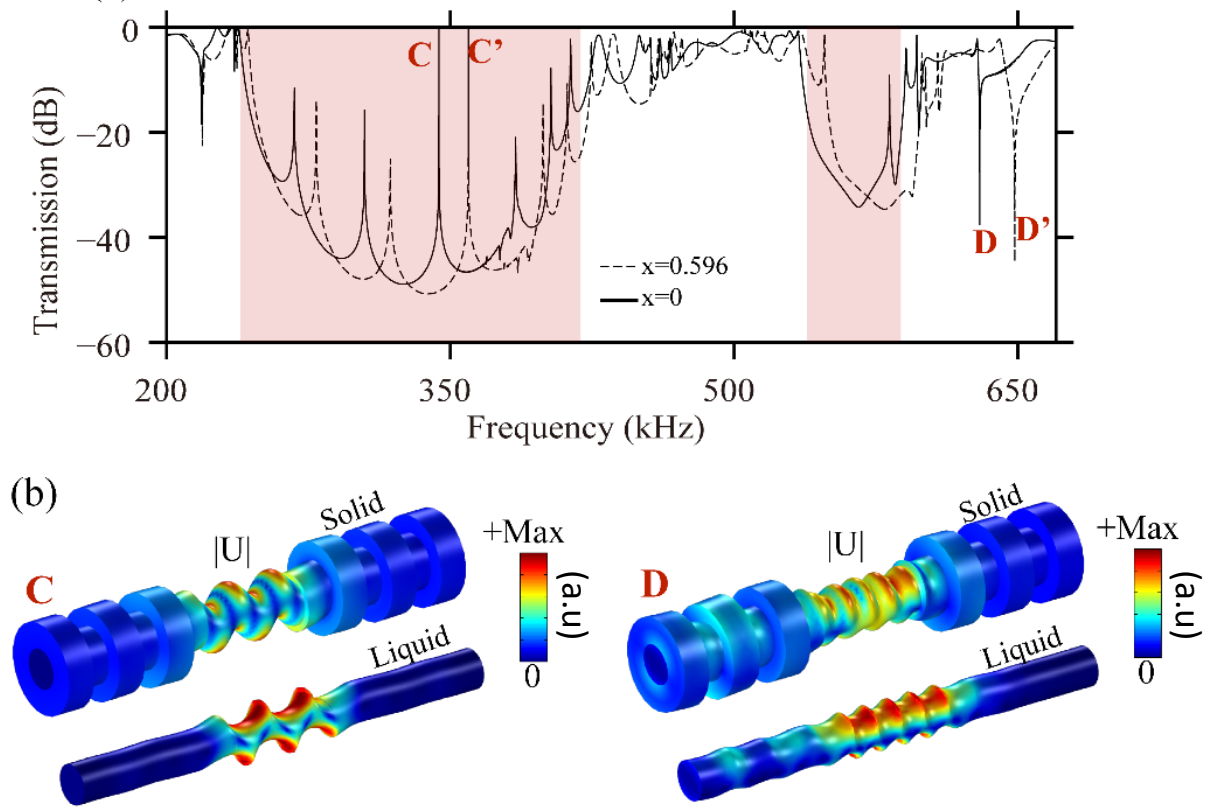

(c)
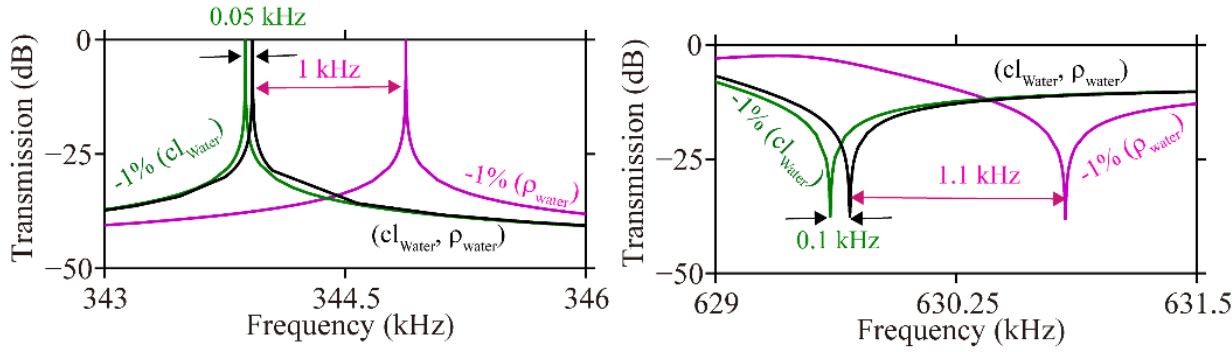

(d)
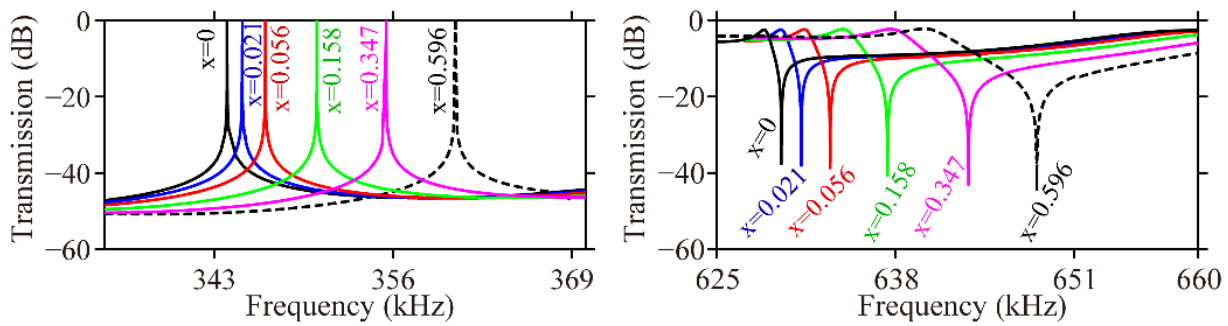

Figure7: (a) Transmission curves of the TPC containing the F-P cavity for a flexural polarization, filled with water (solid line) or a mixture of water and 1-propanol at $x=59.6 \%$ (dashed line). The red areas correspond to the partial band gaps. (b) Maps of the displacement field at the frequencies of peak $\mathrm{C}$ and dip $\mathrm{D}$ in the solid (left panel) and in the fluid (right panel). (c) Shift of the peak C (left panel) and dip D (right panel) when assuming either a change in mass density (pink curves) or the sound velocity (green curves) of water by $1 \%$. Similar to Fig. 5, the sensitivity is much larger for the liquid density. (d) Same as (c) for the set of propanol-water mixtures with molar ratio ranging from 0 to $59.6 \%$. 
The characteristic properties of the transmitted signals are illustrated in Fig. 8 analog to Fig. 6 and summarized in Table 3.
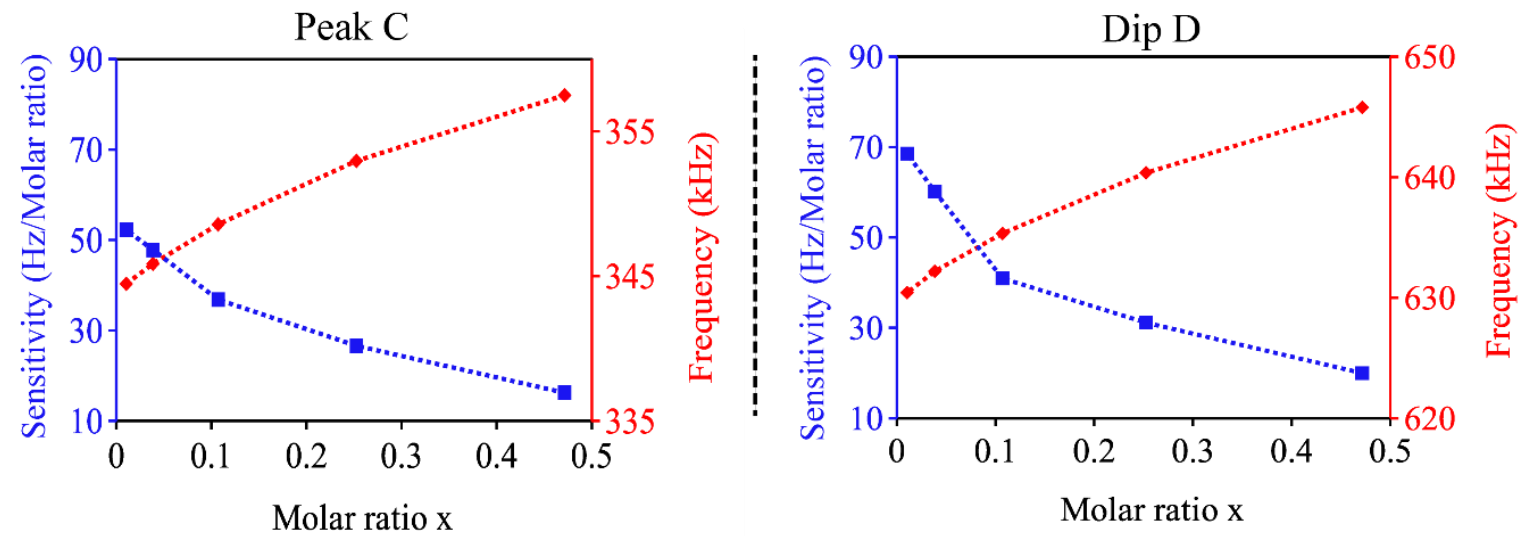

Figure 8: Sensitivity and frequency of the peak C (left panel) and the dip D (right panel) as a function of the molar ratio of the 2-propanol in water.

Table 3: Sensor characteristics calculated for peak $\mathrm{C}$ and dip D, flexural polarization

\begin{tabular}{|c|c|c|c|c|}
\hline & \multicolumn{2}{|c|}{ Peak C } & \multicolumn{2}{|c|}{ Dip D } \\
\hline Frequency $(\mathrm{kHz})$ & \multicolumn{2}{|c|}{343.92} & \multicolumn{2}{|c|}{629.69} \\
\hline \multirow[t]{2}{*}{ Sensitivity } & $\mathrm{S}_{\mathrm{c}}\left(\mathrm{Hz} \mathrm{m}^{-1} \mathbf{s}\right)$ & $\mathrm{S}_{\rho}\left(\mathrm{Hz} \mathrm{kg}^{-1} \mathrm{~m}^{3}\right)$ & $\mathrm{S}_{\mathrm{c}}\left(\mathrm{Hz} \mathrm{m}^{-1} \mathrm{~s}\right)$ & $\mathrm{S}_{\rho}\left(\mathrm{Hz} \mathrm{kg}^{-1} \mathrm{~m}^{3}\right)$ \\
\hline & 2.98 & 95.57 & 6.83 & 111.98 \\
\hline Quality factor & \multicolumn{2}{|c|}{$5.7 \times 10^{5}$} & \multicolumn{2}{|c|}{$3 \times 10^{3}$} \\
\hline Figure of Merit & $\mathrm{FoM}_{\mathrm{c}}(\mathrm{m} / \mathbf{s})^{-1}$ & $\operatorname{FoM}_{\rho}\left(\mathrm{kg}^{-1} \mathrm{~m}^{3}\right)$ & $\operatorname{FoM}_{c}(\mathrm{~m} / \mathrm{s})^{-1}$ & $\operatorname{FoM}_{\rho}\left(\mathrm{kg}^{-1} \mathrm{~m}^{3}\right)$ \\
\hline (FoM) & 4.93 & 158 & 0.03 & 0.53 \\
\hline
\end{tabular}

Both longitudinal and transverse excitations provide similar qualitative information in terms of a liquid sensor. A quantitative comparison of the sensing performances for both polarizations in Tables 2 and 3 and in Figs. 6 and 8 shows that the longitudinal polarization produces larger sensitivities and higher FoMs for peak A compared to peak C. Closer inspection reveals a larger gain of $S_{c}$ and $F o M_{c}$ compared to $S_{\rho}$ and $F_{o M}$. Finally, the sensitivity of the longitudinal polarization is larger at low molar ratios. This is not surprising, since speed of sound of the water-propanol mixture has a maximum at about $x=0.056$; $c_{l}$ is the same at appr. $x=0.157$. Speed of sound and density influence the peak frequency in opposite direction, see Figs. 5(c) and 7(c). 
The behavior of the transmission peaks and dips, B and D, is different. The respective numbers in Table 2 and 3 are roughly the same for both modes. Only the slope of peak $\mathrm{C}$ at low $x$ does not show the aforementioned effect of sound velocity.

\section{Effect of liquid viscosity and material dissipation}

The calculations presented in the previous section (without taking account of the liquid viscosity and material dissipation) are helpful to detect the existence of defect modes that are sensitive to the acoustic properties of the liquid such as the mass density and sound velocity. However, the quality factors of the features observed in the transmission spectrum can in practice be dramatically altered by the absorption phenomena, and more particularly the dynamic viscosity of the liquid. The critical role of each viscosity component is discussed below.

To include the effect of viscosity, the motion of the liquid is described by the linearized Navier-Stokes equation

$$
i \omega \rho v=-\nabla p+\nabla \cdot\left(\mu\left[\nabla v+(\nabla v)^{T}\right]-\left[\frac{2}{3} \mu-\mu_{B}\right][\nabla \cdot v] \overleftrightarrow{I} \quad \text { Eq. } 5\right.
$$

where $p$ is the pressure, $v=i /(\omega \rho) \nabla p$ the velocity, $\mu_{B}$ the bulk viscosity and $\mu$ the dynamic viscosity. The values used in the simulations are those of water ${ }^{41}$, namely $\mu_{B}=0.4 \times$ $10^{-3}$ Pa.s and $\mu=8.9 \times 10^{-4}$ Pa.s. Likewise, we include in a second step the dissipation of the solid material constituting the tube of TPC by means of adding an imaginary part to the Young modulus, namely

$$
E^{c}=(1+i \eta) E \quad E q .6
$$

The values used in the calculations are $\eta=0.01$ and $\eta=0.03$ in order to compare with the results of the preliminary experiments on hollow TPCs reported in the Supplementary Information (SI) section.

In the following, we shall focus on the effect of each absorption term on two main features of the transmission spectrum, namely peak A and dip B, discussed in section 3 (Fig. 5). The effect of liquid viscosity on the transmission spectrum around these two frequencies are presented in Fig.9(a). First, by considering only the bulk viscosity $\mu_{B}$, one can observe a decrease in the peak A and dip B by a factor of 24 and 10 respectively, which results in a decrease of their quality factors. Although this is already a quantitatively significant effect, the main alteration results from the shear viscosity term $\mu$. The latter has a first attenuation 
effect on the compressional motion of acoustic waves in the bulk of the liquid which, however, is in the same order or even smaller than the effect of bulk viscosity $\mu_{\mathrm{B}}$. The main mechanism of dissipation is the shear viscous behavior in the vicinity of the solid-liquid interface in the so-called interface layer boundary (of thickness $\delta_{v i s c}=(2 \mu /(\omega \rho))^{0.5}$ ). Indeed, by including the latter mechanism, the features in Fig. 9 drop by a factor of $10^{5}$ and 63 respectively, which means that they totally vanish and cannot be detected in practice. This observation has not been noticed in previous papers dealing with solid-liquid phononic crystals and would suggest the necessity of a new examination of the results existing in the literature. In our case, the very strong effect of the viscosity can be explained by the highquality factors of the original cavity modes which results in a long transit time, or equivalently a large number of round trips, of the acoustic wave in the cavity. Therefore, to make the cavity modes observable for the purpose of liquid characterization, it becomes necessary to decrease their quality factors. This can be achieved by decreasing the number of washers surrounding the cavity on both sides, for instance from 5 (as in Fig. 5) to $2^{15}$. The new results for the transmission spectra are presented in Fig. 9(b). As a first remark, one can note that for the cavity surrounded by only two washers, the effect of the bulk viscosity $\mu_{B}$ on the amplitude of the peak A and the dip B remains insignificant. When taking account of both viscosity terms $\left(\mu_{B}\right.$ and $\left.\mu\right)$, one can observe a decrease of the peak A and dip B by a factor of 9 and 7 respectively, which makes these features weaker, although still observable. Consequently, the quality factors and figures of merit of these features decrease, while their sensitivities remain almost unaltered. Also, one can note that another dip around $570 \mathrm{kHz}$ becomes more attractive than dip B. A secondary effect of the dynamic viscosity is a shift in the resonance frequencies of peak A and dip B with respect to the case without shear viscosity, an effect which has already been noticed in an early work dealing with airborne phononic crystals ${ }^{4242}$, it was explained by a slowing down of the propagation velocity in the dissipative fluid. 
(a)

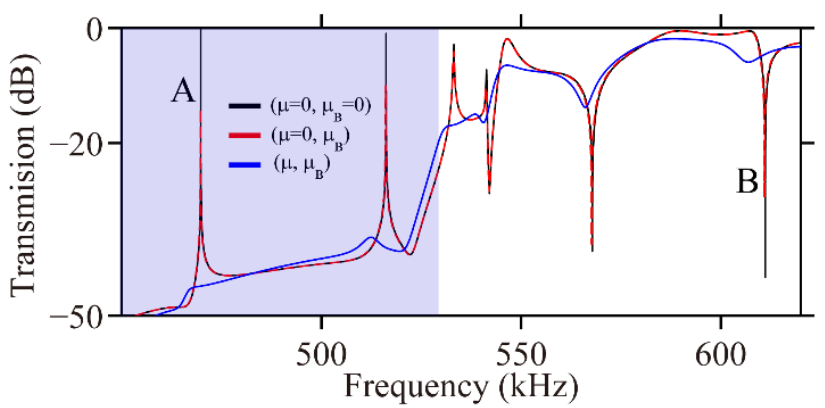

(b)

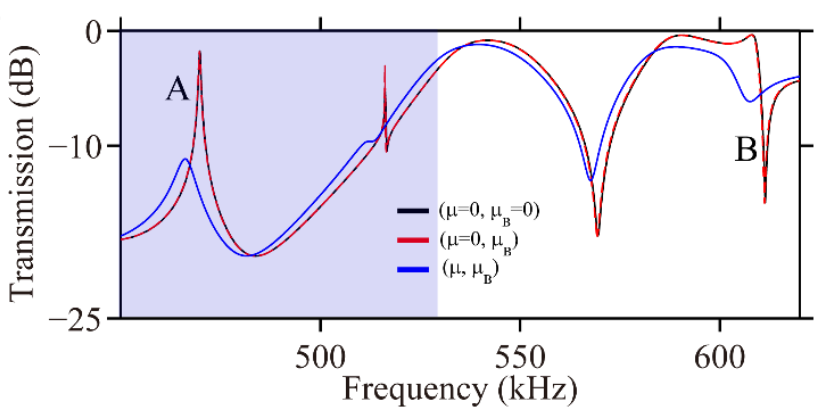

Figure 9: Transmission curves calculated for the TPC with F-P cavity surrounded by (a) 5 washers (b) 2 washers on each side. The black, red and blue curves correspond respectively to the calculated transmission curves without losses, with only $\boldsymbol{\mu}_{B}$ and with both $\boldsymbol{\mu}$ and $\boldsymbol{\mu}_{B}$.

Another possibility to lower the quality factors of the resonances, and hence decrease the absorption phenomena, would be to work at a bigger scale of the phononic crystal and consequently lower frequencies. This can be achieved by multiplying all the geometrical parameters by a same factor.

In the following, we assume that the F-P cavity is surrounded on each side by only two washers and give in Fig. 10 the transmission spectrum when considering the various absorption mechanisms, including the dissipation in the solid material constituting the TPC. As one can see from Fig.10(a), by adding the damping of solid in the calculation, the peak A and the dip B are mitigated by a factor 20 and 9 respectively with an isotropic loss factor of $\eta=0.01$, and 43 and 10 with an isotropic loss factor of $\eta=0.03$. These damping factors were tested as a matter of comparison with the experimental results on hollow TPC samples made of a plastic (see SI). Although the signature of the resonances can still be detected until $\eta=0.03$, one reaches the limit for their observation, which would suggest the necessity of considering less dissipative materials in future TPC samples.

As mentioned in the preceding sections, another alternative for an efficient detection of the cavity peaks is to evaluate the displacement field on the external surface of the cavity, instead of at the exit. The results are presented in Fig. 10(b) where one can remark that in presence of 
both loss mechanisms, the peaks situated in the band gap are more pronounced if measured in the middle of the cavity than at the exit of the TPC. In contrast, the dip B situated in the bulk band is not well pronounced in the middle of the cavity and is visible only at the outlet of the TPC. This finding proves the confinement of acoustic energy inside the cavity even if the observed trends point out the requirement for a TPC material with lower damping.

(a)

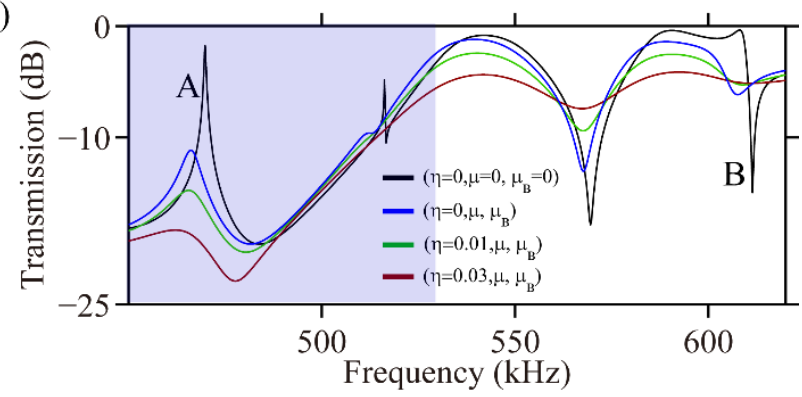

(b)

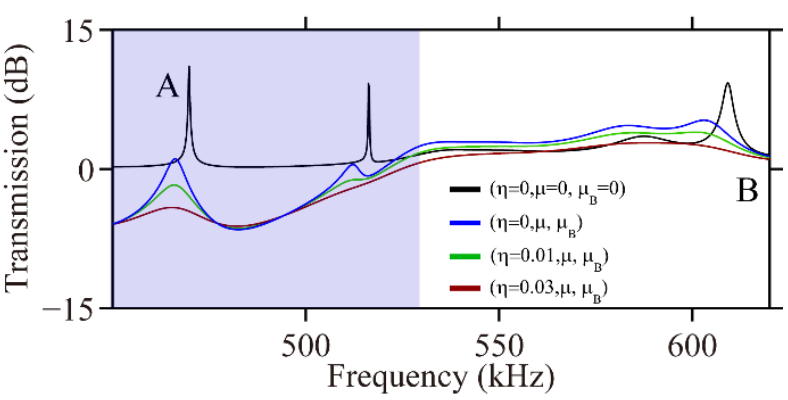

Figure 10: Transmission curves of the TPC with F-P cavity surrounded by two washers on each side where the displacement field is evaluated at (a) the detector region and (b) on the surface of the cavity. The black, blue, green, and brown colors correspond respectively to the calculated transmission curves without losses, with only liquid viscosity and with both liquid viscosity and solid damping when $\boldsymbol{\eta}=\mathbf{0 . 0 1}$ (green) and $\boldsymbol{\eta}=\mathbf{0 . 0 3}$ (brown).

\section{Conclusions}

We have investigated a TPC structured with a periodic arrangement of washers along the tube. We have demonstrated that the mixed solid/liquid system can present absolute or polarization dependent band gaps. The introduction of a Fabry-Perot (F-P) cavity inside the periodic structure leads to the occurrence of peaks (inside the band gaps) and dips (inside the passbands) in the transmission spectrum, which have been shown to be sensitive to the density and speed of sound of the fluid flowing inside the pipe. This result is the consequence of a sufficiently strong coupling of the F-P modes at the fluid/solid interface. The TPC becomes an innovative platform for sensing applications. The above features display higher sensitivities to the variations of the mass density than the sound velocity, with values above $100 \mathrm{~Hz} \mathrm{~kg}^{-1} \cdot \mathrm{m}^{3}$ in our millimeter's size structure. An alternative detection possibility was proposed, namely 
the measurement of the amplitude at the external surface of the cavity. After characterizing the resonances of the F-P cavity with non-viscous liquids, we have discussed the effect of both bulk and shear viscosities of the liquid on these features and shown how to decrease their quality factors to make them observable despite the effect of viscosity. We have also shown that the sensing performances decrease with damping characteristics of the solid material. In the case of polymer used for the tested samples in SI, the comparison between simulations and experimental transmission measurements show a loss coefficient $\eta$ of the order of 0.03 , which could be a limit for its utilization in the TPC sensor. Hence, other materials need to be tested in the future to improve the efficiency of the TPC sensor. The proposed device would be a useful platform for being incorporated in various pipes transporting liquids to be sensed at different scale, from microfluidic to medical or civil engineering applications.

\section{Supplementary Material}

The supplementary information section gives some preliminary experiments, before liquid filling, with a few samples of TPC fabricated with the help of a 3D printer.

\section{Acknowledgements:}

This work was performed in the frame of the project 'Tubular Bell' supported by the national French agency ANR-18-CE92-0023 and by the Deutsche Forschungsgemeinschaft (DFG, German Research Foundation) undergrants VE 483/2-1 and LU 605/22-1.

Data Availability:

The data that support the findings of this study are available from the corresponding author up on reasonable request. 


\section{Appendix}

Figure 13 shows the evolution of the dispersion curves of the water-filled tubular phononic crystal as a function of the geometrical parameters. The optimal parameters leading to the largest absolute band gap are $r=0.3 \times a, w=0.5 \times a$ and $h=0.3 \times a$, with a $=1 \mathrm{~mm}$.
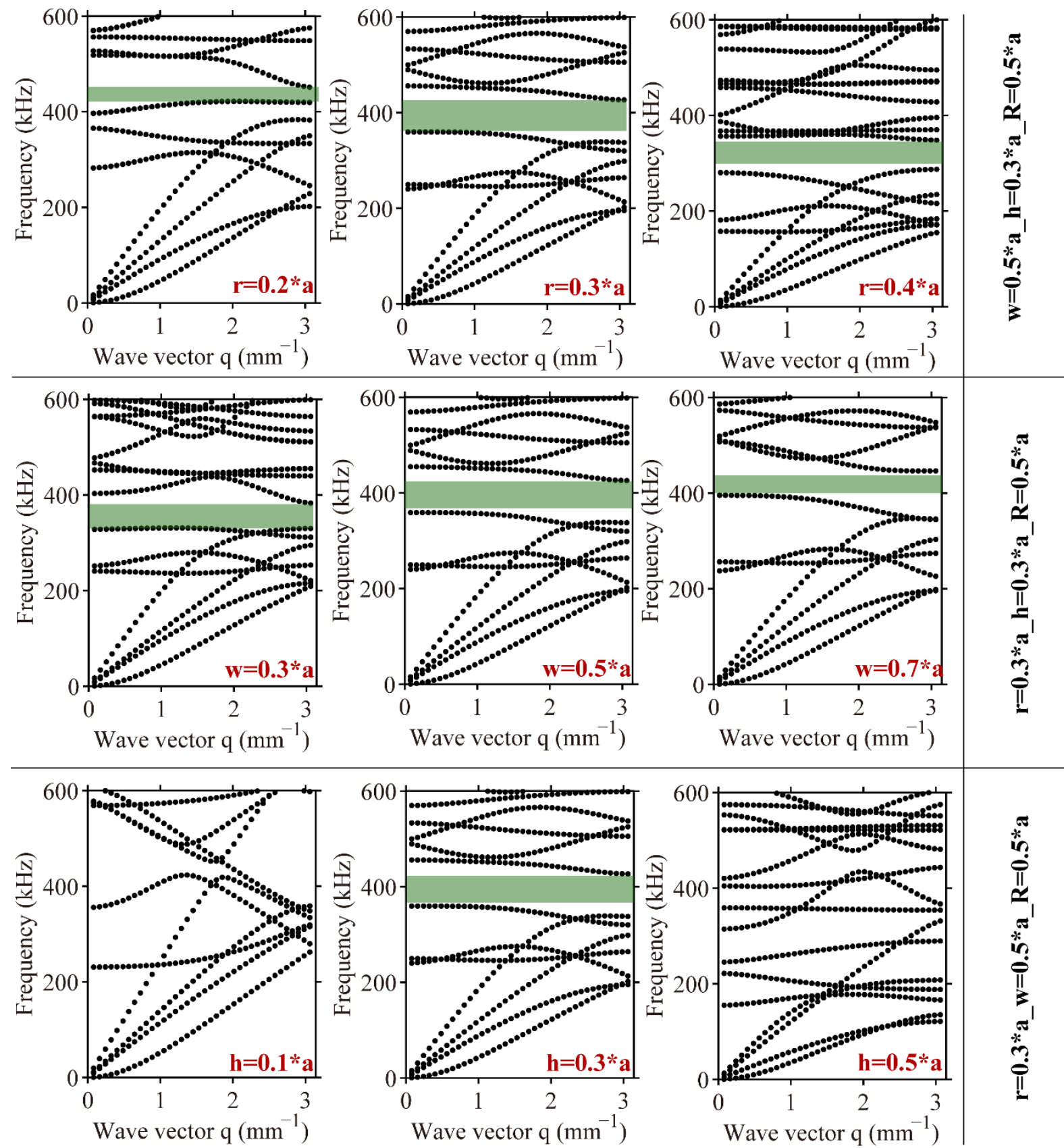

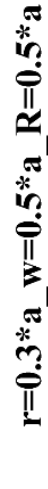

Figure 13: Evolution of the dispersion curves of the water-filled TPC as a function of the geometrical parameters; the green area represents the phononic band gap. 


\section{References}

${ }^{1}$ M.S. Kushwaha, P. Halevi, L. Dobrzynski, and B. Djafari-Rouhani, Phys. Rev. Lett. 71, 2022 (1993).

${ }^{2}$ A. Khelif and A. Adibi, editors, Phononic Crystals: Fundamentals and Applications (Springer-Verlag, New York, 2016).

${ }^{3}$ V. Laude, Phononic Crystals: Artificial Crystals for Sonic, Acoustic, and Elastic Waves (Walter de Gruyter GmbH \& Co KG, 2015).

${ }^{4}$ Y. Pennec, J.O. Vasseur, B. Djafari-Rouhani, L. Dobrzyński, and P.A. Deymier, Surface Science Reports 65, 229 (2010).

${ }^{5}$ Y. Pennec, Y. Jin, and B. Djafari-Rouhani, in Advances in Applied Mechanics, edited by M.I. Hussein (Elsevier, 2019), pp. 105-145.

${ }^{6}$ F. Lucklum, Meas. Sci. Technol. 32, 085108 (2021).

${ }^{7}$ A.A. Shehatah and A. Mehaney, Mater. Res. Express 6, 125556 (2020).

${ }^{8}$ A. Oseev, N. Mukhin, R. Lucklum, M. Zubtsov, M.-P. Schmidt, U. Steinmann, A. Fomin, A. Kozyrev, and S. Hirsch, Sensors and Actuators B: Chemical 257, 469 (2018).

${ }^{9}$ A. Sato, Y. Pennec, N. Shingne, T. Thurn-Albrecht, W. Knoll, M. Steinhart, B. DjafariRouhani, and G. Fytas, ACS Nano 4, 3471 (2010).

${ }^{10}$ K.Keiji. Kanazawa and J.G. Gordon, Anal. Chem. 57, 1770 (1985).

${ }^{11}$ B.O. Alunda and Y.J. Lee, Sensors (14248220) 20, 4784 (2020).

${ }^{12}$ Y.T. Yang, C. Callegari, X.L. Feng, K.L. Ekinci, and M.L. Roukes, Nano Lett. 6, 583 (2006).

${ }^{13}$ R. Kazys, R. Sliteris, R. Raisutis, E. Zukauskas, A. Vladisauskas, and L. Mazeika, Appl. Phys. Lett. 103, 204102 (2013).

${ }^{14}$ F. Simonetti, P. Cawley, and A. Demčenko, The Journal of the Acoustical Society of America 118, 832 (2005).

${ }^{15}$ R. Lucklum and J. Li, Meas. Sci. Technol. 20, 124014 (2009).

${ }^{16}$ S. Villa-Arango, R. Torres, P.A. Kyriacou, and R. Lucklum, Measurement 102, 20 (2017).

${ }^{17}$ F. Khateib, A. Mehaney, R.M. Amin, and A.H. Aly, Phys. Scr. 95, 075704 (2020).

${ }^{18}$ R. Lucklum, M. Ke, and M. Zubtsov, Sensors and Actuators B: Chemical 171-172, 271 (2012).

${ }^{19}$ S. Amoudache, Y. Pennec, B. Djafari Rouhani, A. Khater, R. Lucklum, and R. Tigrine, Journal of Applied Physics 115, 134503 (2014).

${ }^{20}$ H. Gharibi, A. Mehaney, and A. Bahrami, J. Phys. D: Appl. Phys. 54, 015304 (2020).

${ }^{21}$ A. Cicek, D. Trak, Y. Arslan, N. Korozlu, O.A. Kaya, and B. Ulug, ACS Sens. 4, 1761 (2019).

${ }^{22}$ R. Lucklum, M. Zubtsov, and M. Ke, in 2011 Joint Conference of the IEEE International Frequency Control and the European Frequency and Time Forum (FCS) Proceedings (2011), pp. 1-4.

${ }^{23}$ M. Ke, M. Zubtsov, and R. Lucklum, Journal of Applied Physics 110, 026101 (2011).

${ }^{24}$ S.E. Zaki, A. Mehaney, H.M. Hassanein, and A.H. Aly, Scientific Reports 10, 17979 (2020).

${ }^{25}$ S. Amoudache, R. Moiseyenko, Y. Pennec, B.D. Rouhani, A. Khater, R. Lucklum, and R. Tigrine, Journal of Applied Physics 119, 114502 (2016).

${ }^{26}$ Y. Jin, Y. Pennec, Y. Pan, and B. Djafari-Rouhani, Crystals 6, 64 (2016).

${ }^{27}$ T.-T. Wang, Y.-F. Wang, Y.-S. Wang, and V. Laude, Appl. Phys. Lett. 111, 041906 (2017).

${ }^{28}$ J. Bonhomme, M. Oudich, B. Djafari-Rouhani, F. Sarry, Y. Pennec, B. Bonello, D.

Beyssen, and P.G. Charette, Appl. Phys. Lett. 114, 013501 (2019). 
${ }^{29}$ R. Lucklum, M. Zubtsov, and A. Oseev, Anal Bioanal Chem 405, 6497 (2013).

${ }^{30}$ N.-N. Huang, Y.-C. Chung, H.-T. Chiu, J.-C. Hsu, Y.-F. Lin, C.-T. Kuo, Y.-W. Chang, C.Y. Chen, and T.-R. Lin, Crystals 10, 421 (2020).

${ }^{31}$ T.-X. Ma, Y.-S. Wang, C. Zhang, and X.-X. Su, Sensors and Actuators A: Physical 242, 123 (2016).

${ }^{32}$ G. Sharma, S. Kumar, and V. Singh, Acoustical Physics 63, 159 (2017).

${ }^{33}$ R. Lucklum, M. Zubtsov, and Y. Pennec, Procedia Engineering 120, 520 (2015).

${ }^{34}$ H. Shen, J. Wen, D. Yu, and X. Wen, Journal of Sound and Vibration 328, 57 (2009).

${ }^{35}$ J. Plisson, A. Pelat, F. Gautier, V.R. Garcia, and T. Bourdon, Appl. Phys. Lett. 116, 201902 (2020).

${ }^{36}$ T. Liu, L.-L. Xu, H. Liu, Y.-X. Fan, and Z.-Y. Tao, AIP Advances 10, 095109 (2020).

${ }^{37}$ J. Jung, C.-H. Jeong, and J.S. Jensen, Appl. Phys. Lett. 115, 041903 (2019).

${ }^{38}$ M. Xiao, G. Ma, Z. Yang, P. Sheng, Z.Q. Zhang, and C.T. Chan, Nature Phys 11, 240 (2015).

${ }^{39}$ C.J. Burton, The Journal of the Acoustical Society of America 20, 186 (1948).

${ }^{40} \mathrm{~J}$. Li and J.L. Rose, IEEE Transactions on Ultrasonics, Ferroelectrics, and Frequency Control 49, 1720 (2002).

${ }^{41}$ A.S. Dukhin and P.J. Goetz, J. Chem. Phys. 130, 124519 (2009).

${ }^{42}$ A.O. Krushynska, F. Bosia, and N.M. Pugno, ArXiv:1712.06063 [Cond-Mat, Physics:Physics] (2017). 